\title{
Disaster Monitoring by Extracting Geophysical Parameters from SAR Data
}

\author{
Gerardo Di Martino, Antonio Iodice, Daniele Riccio, Giuseppe Ruello \\ Dipartimento di Ingegneria Elettronica e delle Telecomunicazioni, \\ Università di Napoli "Federico II" \\ Via Claudio 21, 80125 Napoli, Italy \\ E-mail: \{gerardo.dimartino, iodice, daniele.riccio, ruello\}@unina.it
}

\begin{abstract}
The fractal geometry proved to be the most appropriate mathematical instrument in describing natural scenes, by means of few effective and reliable geophysical parameters. In this paper we use fractal concepts to model and to identify geometrical changes occurred in areas hit by disasters. We present an overall framework employing fractal based models, algorithms and tools to support identification of natural area changes due to natural or man-made disasters. The proposed framework includes a SAR raw signal simulator, which is of key importance to improve the comprehension of the mechanisms underlying SAR image formation. In addition, we consider, as a case study, the simulation and detection of lava flows in a volcanic scenario. The potentialities of our technique for the discrimination between different types of lava are presented and discussed.
\end{abstract}

Keywords - Synthetic Aperture Radar, Fractals, Disaster Monitoring

\section{INTRODUCTION}

Natural disasters can be catastrophic for human beings, especially if a reliable response is not quickly organized. Remote sensing tools are extremely important for the definition of such a response because they provide a huge amount of data relevant to almost any area of the Earth.

Several approaches devoted to define instruments and tools for data interpretation were presented in literature [1]. Most of them are based on empirical analyses of remote sensing data, essentially driven by user needs. These analyses are generally supervised and, to be effective, it is often required that the supervisor holds a remarkable level of competence with reference both to the sensors, and to the effects of different disasters on the environment.

In this paper, we focus our attention on SAR sensors, because the microwave frequencies employed by radar instruments and the obtained geometric resolutions are better tailored to exploit the geometrical features of the area under survey. Furthermore, SAR all-weather, all-time characteristics are of fundamental importance for disaster observation purposes. Anyway, the difficulty in interpretation of this kind of data often limits their use to expert observers.

When a disaster occurs, the observed scene dramatically changes, and remote sensing instruments should be, at least in principle, able to detect these changes. The natural disasters modify (according to different rules) the surface profile from scales smaller than the sensor coverage but comparable to the sensor resolution, up to scales comparable to the electromagnetic wavelength.

To develop unsupervised or semi-unsupervised tools for the extraction of added value information from remotely sensed images it is crucial to introduce appropriate models to understand and quantitatively describe the physical phenomena governing the modification of the scenario textures.

We suggest the combined use of direct and inverse fractal models. In this paper, we present an overall framework in which these element are combined into an appropriate elaboration chain.

As for the direct models, the fractal geometry [2] has the required characteristics to manage the problem at hand, because it simply accounts for the complexity of the observed objects, by means of few effective geophysical parameters. In Section II, we outline our framework and present the above mentioned direct and inverse models. Note that a more detailed discussion on the algorithms and tools used in our elaboration chain can be found in [10].

As far as the inverse models are under concern, they are used to retrieve SAR image fractal parameters and for change detection purposes. In particular, the comparison between preand post- crisis SAR image fractal parameters and the determination of the fractal dimension of the contour of the region of interest provide value added information for change detection purposes, as detailed in Section III.

In Section IV, we test the fractal framework in a simulated disaster scenario. In particular, we simulate lava flows with fractal contour and show the performance of our fractal change detection algorithm. In addition, an analysis of lava flow borders, performed on SAR simulated images and aimed at discriminating different types of lava, is presented.

\section{DIRECT MODELS}

In this Section we present the fractal framework and we outline the rationale of the proposed method. 


\section{A. SAR simulation}

In past years, a SAR raw signal simulator was developed and tested [3], [4]. A SAR data simulator is an important tool to help scientists in better understanding the mechanisms underlying SAR image formation and in the interpretation of SAR data textures and of their modifications.

In the following we describe briefly the key issues for SAR signal simulation. Let $x$ and $r$ be the independent space variables, standing respectively for azimuth and range. By using primed coordinates for the independent variables of the SAR raw signal, $s\left(x^{\prime}, r^{\prime}\right)$, this can be expressed as [3]:

$$
s\left(x^{\prime}, r^{\prime}\right)=\iint d x d r \gamma(x, r) g\left(x^{\prime}-x, r^{\prime}-r ; r\right),
$$

where $\gamma(x, r)$ is the reflectivity pattern of the scene and $g\left(x^{\prime}-x, r^{\prime}-r ; r\right)$ the unit impulse response of the SAR system [3], [4]. Evaluation of the reflectivity pattern requires a description of the observed surface as well as a model for their interaction with the electromagnetic fields radiated by the SAR antenna [3]. In this paper we use the models presented in the next two paragraphs.

\section{B. Fractal surface model}

Fractal models are widely considered the most appropriate to quantitatively describe natural surfaces [2]. Fractal geometry is able to simply account for the non-stationarity of natural surfaces, as well as for their self-affinity. The most used fractal model is the fractional Brownian motion $(\mathrm{fBm})$ [5]. The $\mathrm{fBm}$ is defined in terms of the probability density function of its height increments: a stochastic process $z(x, y)$ is an $\mathrm{fBm}$ surface if, for every $x, y, x^{\prime}, y^{\prime}$, it satisfies the following relation:

$$
\operatorname{Pr}\left\{z(x, y)-z\left(x^{\prime}, y^{\prime}\right)<\bar{\zeta}\right\}=\frac{1}{\sqrt{2 \pi} s \tau^{H}} \int_{-\infty}^{\bar{\zeta}} \exp \left(-\frac{\zeta^{2}}{2 s^{2} \tau^{2 H}}\right) d \zeta
$$

where $\tau$ is the distance between the points $(x, y)$ and $\left(x^{\prime}, y^{\prime}\right)$, and the two parameters that control the $\mathrm{fBm}$ behaviour are:

$H$ : the Hurst coefficient $(0<H<1)$, related to the fractal dimension $D$ by means of the relation $D=3-H$.

$s:$ the standard deviation, measured in $\left[\mathrm{m}^{(1-H)}\right]$, of surface increments at unitary distance, a real parameter related to an $\mathrm{fBm}$ characteristic length, the topothesy $T$, by means of the relation $s=T^{(1-H)}$.

To compute the reflectivity function of the observed scene the considered simulator requires as input a DEM relative to the scene of interest, sampled with a resolution coherent with the considered sensor parameters: in practice, we need to interpolate the available DEM [4].

We use the $\mathrm{fBm}$ to model the surface imaged by the SAR sensor. Hence, the fractal parameters, retrieved from the considered DEM, are used to perform a stochastic fractal interpolation on the available DEM. In this way, the interpolated DEM inherits the fractal behaviour of the original surface [4].

\section{Fractal scattering model}

Theoretical [2] and experimental [7] studies suggest that use of fractal models improve the scattering method results. In this paper we use the $\mathrm{fBm}$ fractal model in describing the surface roughness and the small perturbation method (SPM) as scattering model for evaluating the reflectivity pattern [6].

Comparison between simulated and actual SAR data was presented in [4] with respect to image single point normalised moments and autocorrelation function, thus assessing the simulator reliability. In those comparisons the fractal parameters accounting for the microscopic description of the scene were assumed to be constant. In this case the extension we propose allows considering for the microscopic scale (up to the electromagnetic wavelength scale) the fractal parameters estimated from the available DEM, so that in order to compute the reflectivity function we use fractal parameters varying all over the scene of interest [10].

\section{Results}

In order to show the ability of the presented simulator for reproducing SAR data, we show some meaningful simulation results. As an example, we provided as input for the simulator the digital elevation model (DEM) of Kilimanjaro volcano $\left(2^{\circ} \mathrm{S}\right.$ $36^{\circ} \mathrm{E}$ ), provided on a $90 \times 90 \mathrm{~m}^{2}$ grid. The DEM was interpolated via the fractal approach introduced in [10], in order to be described on a rectangular grid, with pixel dimensions of $3.99 \times 19.9 \mathrm{~m}^{2}$, in accordance with the ERS-1 acquisition geometry. In Fig. 1 we present a 3D representation of the DEM.

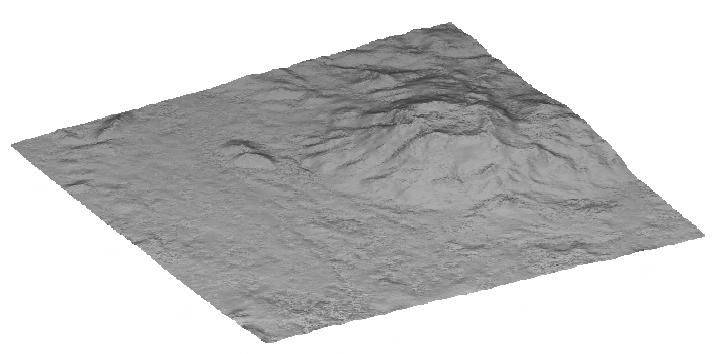

Figure 1. 3D representation of the Kilimanjaro volcano area.

The microscopic roughness, responsible for the electromagnetic scattering, is provided by estimating the local fractal dimension of the DEM. Therefore, we simulated the SAR image acquired by the ERS- 1 sensor. The dielectric constant $\varepsilon$ of the scene is set to $\varepsilon=4 \varepsilon_{0}$, and the conductivity $\sigma$ is set to $\mathrm{s}=0.001 \mathrm{~S} / \mathrm{m}$.

The simulated image is presented in Fig. 2 (near range is on the left). The SAR image was obtained from the simulated raw signal via standard processing and averaged with a $2 \times 10$ multi-look, in order to obtain an azimuth-ground range approximately square pixel. Such an image represent a 
reference for the situation, where it is assumed that the lava is not present in the scene

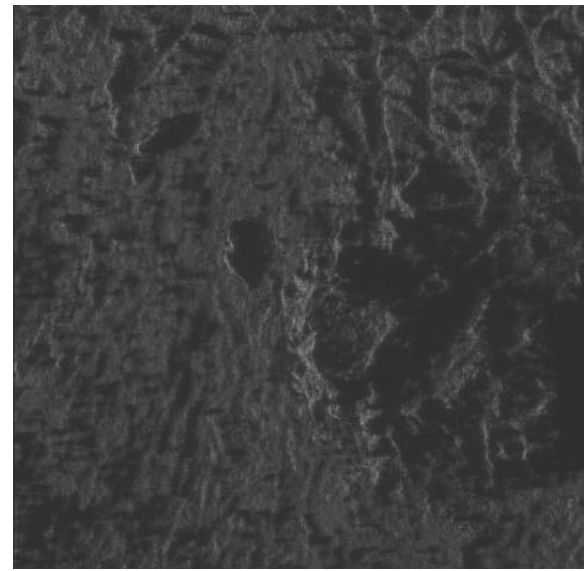

Figure 2. Simulated ERS-1 image of the Kilimanjaro area. Near range is on the left.

\section{INVERSE MODELS}

In the open literature, several methods for the extraction of information from SAR images relative to scenarios subject to changes are proposed. The majority of them are based on classical magnitude change detection techniques such as ratioing and differencing [8].

In this paper we present a technique for the extraction of fractal parameters, whose use can bring value added information. In particular, we propose a twofold extraction of fractal parameters, one based on the intensity variation, the other on the extraction of the fractal parameters of a region of interest (ROI) contour.

\section{A. SAR image fractal dimension extraction}

An analytical relationship between the fractal dimension of a portion of surface and the fractal dimension of the corresponding SAR image is still unknown. Anyway, the use of the SAR simulator allows the definition of a wide set of canonical scenes, which can provide an empirical relatiponship [10].

Such a result can be reached if an appropriate fractal dimension extraction technique is applied. For a given surface, the structure function (variogram), $V(\tau)$, is defined as the mean square increment of elevation points placed at distance $\tau$ :

$$
V(\tau)=\left\langle\left(z(x, y)-z\left(x^{\prime}, y^{\prime}\right)\right)^{2}\right\rangle
$$

The variogram of an $\mathrm{fBm}$ surface can be evaluated in terms of the parameters $H$ and $s$ and expressed in logarithmic form as:

$$
\log V(\tau)=2 \log s+2 H \log \tau,
$$

which defines in a log-log plane a linear behaviour with slope $2 H$, and ordinate intercept $2 \log s$. Such a dependence leads to retrieve the fractal parameters with a linear regression over the $\log -\log$ plots of measured values of $V(\tau)$ [6].

Note that the application of this technique to SAR images is someway critical, because it is necessary to take into account the non equal spacing of the data set. In this work we enlarged the available technique validity to deal with such a case.

\section{B. Extraction of the ROI contour fractal dimension}

In several applications, different regions of interest can be classified in accordance with the roughness of their contour. To this aim, a box counting algorithm can be employed, provided that the region of interest was previously defined.

This operation can be not straightforward on SAR images due to the presence of speckle. Therefore, a pre-processing step, possibly based on the techniques presented in the previous section, is required. We exploited here the simulator ability of generating a training set for understanding the technique performances and limitation. As an example, we generated two areas of different contour fractal dimension, as presented in Fig. 3. The left and the right areas have a contour fractal dimensions of $D=1.27$, and $D=1.3$, respectively.
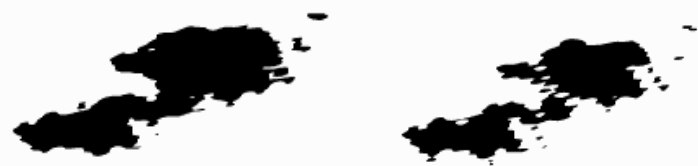

Figure 3. Simulated areas with fractal dimension $D=1.27$ (on the left) and $D=1.3$ (on the right).

These masks can be used to define areas with different geometrical (fractal dimension and topothesy) and dielectric (permittivity and conductivity) parameters with respect to an homogeneous background. Then, we estimated the corresponding area (via the techniques presented in the previous section) in the SAR image and we obtained that the their contour fractal dimensions $D_{S}$ are: $D_{S}=1.23$ and $D_{S}=1.27$, respectively. As expected the fractal dimension is almost the same. An underestimation is mainly due to the filtering of the speckle necessary to extract the area from the SAR image.

\section{THE CASE STUdy}

In the following, the potentiality of the chain presented in the previous section is tested on a case study, focused on the monitoring of volcanic areas. In particular, a novel approach is proposed, based on change detection techniques applied to fractal parameters retrieved from pre- and post-crisis images.

The region of interest is the area of the Kilimanjaro volcano, presented in Section II. In order to estimate the ability of the proposed technique, we simulated a lava eruption, by setting the simulation parameters of a given region to the typical values for the most common lava flows (AA and pahoehoe).

In order to appropriately account for the presence of lava, we gave the region parameters the values defined in Table I. 
Two kinds of lava are defined: a smoother one, recalling the properties of the pahoehoe lava flows and a rougher one, recalling the properties of the AA lava flows. In Fig. 4 and Fig. 5 the two simulated SAR images are presented.

TABLE I. SAR SENSOR PARAMETERS

\begin{tabular}{|l|c|c|}
\hline \multicolumn{1}{|c|}{ Lava parameters } & AA & Pahoehoe \\
\hline Dielectric Constant & 8 & 20 \\
\hline Conductivity $[\mathrm{S} / \mathrm{m}]$ & 0.01 & 1 \\
\hline Hurst coefficient & 0.6 & 0.85 \\
\hline $\left.\mathrm{s}\left[\mathrm{m}^{\wedge}(1-H)\right)\right]$ & 0.1 & 0.2 \\
\hline
\end{tabular}

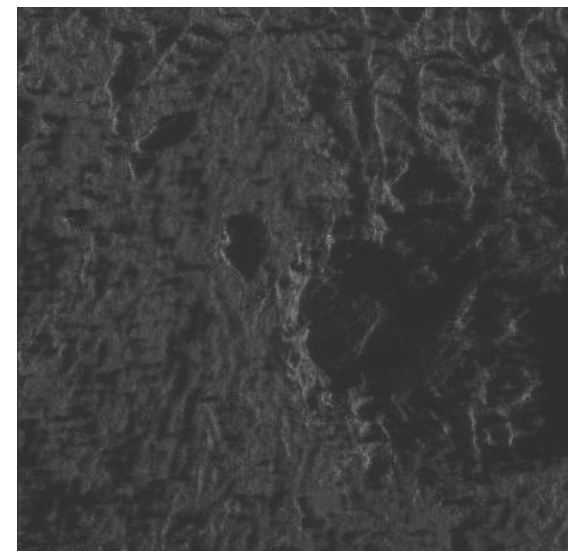

Figure 4. Simulated ERS-1 image of the Kilimanjaro area. The area surrounding the crater is covered by pahoehoe lava. Near range is on the left.

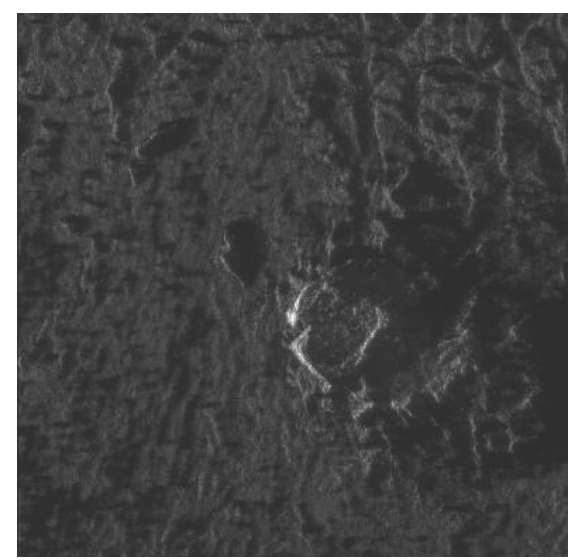

Figure 5. Simulated ERS-1 image of the Kilimanjaro area. The area surrounding the crater is covered by AA lava. Near range is on the left.

On the obtained SAR images we applied the variogram method, obtaining the maps of the fractal parameters of the two scenarios for comparison purposes. By comparing the image intensity, as well as the fractal parameter values, the region covered by the lava can be extracted.

In order to quantify the performance of the technique, a reference map can be generated, by simulating the SAR data in absence of speckle in the pre- and post- eruption cases. Their difference defines the "ground truth" for the case study.
In Fig. 6 and Fig. 7 the ground truth and the map obtained with the proposed approach are shown.
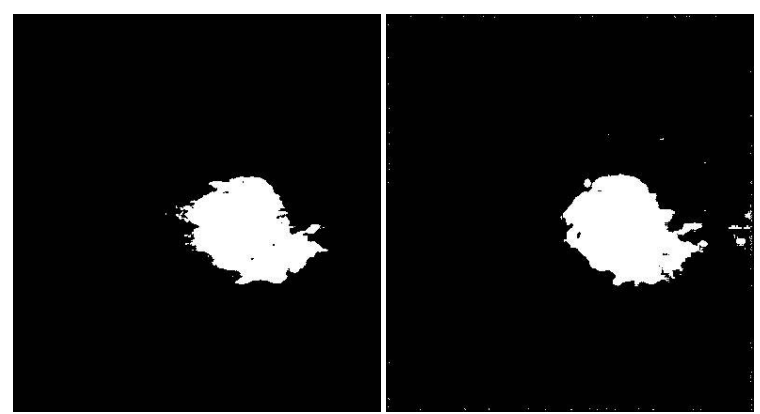

Figure 6. Comparison between ground truth map (on the left) and the classification obtained with the proposed technique (on the right).

\section{CONCLUSIONS}

In this paper we presented a complete framework of fractal models whose use allows to improve the comprehension, interpretation, and information extraction from SAR images. A case study was faced, regarding a volcano area. The presence of lava flows was simulated, with the goal of understanding the SAR data formation mechanisms and improving the parameter retrieving algorithms. The obtained results show that the proposed techniques lead to a significant performances in the extraction of information from SAR data.

\section{REFERENCES}

[1] P. Lundgren, P. Berardino, M. Coltelli, G. Fornaro, R. Lanari, G. Puglisi, E. Sansosti and M. Tesauro, "Coupled Magma Chamber Inflation and Sector Collapse Slip Observed with Synthetic Radar Interferometry on Mt. Etna", Journal of Geophysical Research, 108, n. B5, pp. 2247-2262, 2003.

[2] B.B. Mandelbrot, The Fractal Geometry of Nature, W.H.Freeman \& C., New York, USA, 1983.

[3] G. Franceschetti, M. Migliaccio, D. Riccio, G. Schirinzi, "SARAS: a SAR Raw Signal Simulator", IEEE Trans. Geosci. Remote Sens., vol. 30, no. 1, pp. 110-123, Jan.1992.

[4] G. Franceschetti, M. Migliaccio, D. Riccio, "SAR Raw Signal Simulation of Actual Scenes Described in Terms of Sparse Input Data", IEEE Trans. Geosci. Remote Sens., vol. 32, no. 6, pp. 1160-1169, Nov. 1994.

[5] G. Franceschetti, A. Iodice, M. Migliaccio, D. Riccio, "Scattering from Natural Rough Surfaces Modelled by Fractional Brownian Motion TwoDimensional Processes", IEEE Trans. Antennas Prop., vol. 47, no. 9, pp. 1405-1415, Sept.1999.

[6] G. Franceschetti, A. Iodice, D. Riccio, "Fractal Models for Scattering from Natural Surfaces", Invited Chapter in Scattering, R. Pike, P. Sabatier, eds., Academic Press, London (UK), pp. 467-485, Sept. 2001.

[7] G. Ruello, P. Blanco, A. Iodice, J. J. Mallorqui, D. Riccio, A. Broquetas, G. Franceschetti, "Synthesis, Construction And Validation Of A Fractal Surface" IEEE Trans. Geosci. Remote Sens., vol. 44, no. 6, pp. 14031412, June 2006.

[8] E. Rignot and J. van Zyl, "Change Detection Techniques for ERS-1 SAR Data”, IEEE Trans. Geosci. Remote Sens., vol. 31, no. 4, pp.896906, July 1993.

[9] F. T. Ulaby, R. K. Moore and A. K. Fung, Microwave Remote Sensing: Active and Passive, Reading, MA: Addison-Wesley, 1982.

[10] G. Di Martino, A. Iodice, D. Riccio and G. Ruello, “A Novel Approach for Disaster Monitoring: Fractal Models and Tools", IEEE Trans. Geosci. Remote Sens., in press. 